\title{
CONCERNING INDIVIDUAL DIFFERENCES IN REACTION TIMES
}

\author{
BY V. A. C. HENMON \\ University of Wisconsin, Madison, Wis. \\ AND \\ F. LYMAN WELLS \\ McLean Hospital, Waverley, Mass.
}

The fact of marked individual differences in reaction times and in types of reaction has often been noted. Less frequently has the question been raised as to the persistence of these differences after long-continued practice. Still less frequently if ever has the correlation between the times of simple and compound reactions been especially treated.

On the persistence of differences after practice, Alechsieff ${ }^{1}$ says, "Individual differences in transit observations have been especially referred in part to practice and in part to the employment of different modes of reaction.... If the observers are thoroughly practised, personal differences become vanishingly small." Similarly, Whipple ${ }^{2}$ says, "And now most important is this: when practice is attained, as indicated by a mean variation less than 10 per cent. of the average or median value, the results judging from my experience in the laboratory at least, are practically identical for all observers. In other words, our constant individual differences have disappeared since they themselves depend upon the degree of practice and upon variation in the direction of attention." These statements do not agree with the writers' experience with reaction time measurements. Individual differences both in simple and compound reactions persist after very long periods of practice and rest on more fundamental differences than those

1 Alechsieff, 'Reactionszeiten bei Durchgangsbeobachtungen,' Philosophische Studien, 16, 1900, pp. 26-27.

2 Whipple, G. M., 'Reaction-Times as a Test of Mental Ability,' Am. Jour. of Psychol., Vol. XV., 1904, Pp. 489-498. 
of practice and direction of attention. There appears also to be a surprising lack of correlation between the times for simple and compound reactions.

In the study ${ }^{1}$ of the time of perception as a measure of differences in sensations by one of the writers, long series of experiments on differences in color and pitch were made on two subjects, $H$ and $W$. The average times for discriminative or choice reactions to red and yellow ( 440 cases) were as follows: $H$

$W$

av. $216.9 \sigma$, m.v. I7 $7 \sigma \sigma$

av.256.5 $\sigma$, m.v. $22.1 \sigma$

Before taking these series $H$ had made approximately 2,000 simple reactions to sound and light stimuli and 3,000 discriminative or choice reactions to colors for the purpose of eliminating practice effects. $W$ had made similarly a considerable but unknown number of simple reactions and approximately 1,500 compound reactions. From the time that the published experiments with the red and yellow were begun no appreciable differences in the times due to practice were discoverable. Similar differences between the times for the two subjects were shown in the experiments on pitch. The results for the differences of 16 vibrations ( 320 cases) were as follows:

$H$

av. $290.2 \sigma$, m.v. $26.6 \sigma$
$W$

av. $344.3 \sigma$, m.v. $40.9 \sigma$

After the completion of the study from which the above results are drawn $H$ and $W$ began certain studies of reaction times, only a portion of which was ever completed. Before these were begun $W$ had made approximately 5,000 simple reactions (to visual stimuli) and 5,000 compound reactions; $H$ approximately 4,000 simple reactions (visual and auditory) and 14,000 compound reactions. So far as it is possible ever to be sure in such matters practice effects had been entirely eliminated. The high degree of practice which both subjects had attained seemed to furrnish an invaluable opportunity for a study of reaction times in general. The first experiments consisted of $1, \infty 00$ simple muscular reactions to auditory stimuli, the Zimmerman sound hammer being used as stimulator.

1 Henmon, 'The Time of Perception as a Measure of Differences in Sensations,' Archives of Philosophy, Psychology and Seientific Methods, No. 8, 1906. 
Series of 100 reactions were taken each day. The results were as follows:

\section{$H$}

$W$

av. $125.4 \sigma$, m.v. $10.5 \sigma$.

av. I05.3 $\sigma$, m.v. $10.5 \sigma$.

A contemporary research carried out independently in the same laboratory, ${ }^{2}$ afforded data on these same individuals concerning the simple reaction times to visual stimuli of varying sizes. The averages of all reactions taken with each subject were as follows:

$$
\begin{gathered}
H \\
500 \text { reactions, av. } 184.6 \sigma, \text { m.v. } 9.9 \sigma \\
W W \\
\text { 2,000 reactions, av. } \mathrm{I} 73.6 \sigma \text { m.v. } 8.6 \sigma
\end{gathered}
$$

The distribution of the observations in the preceding results with the exception of those for simple visual reactions, is shown graphically in the accompanying chart.

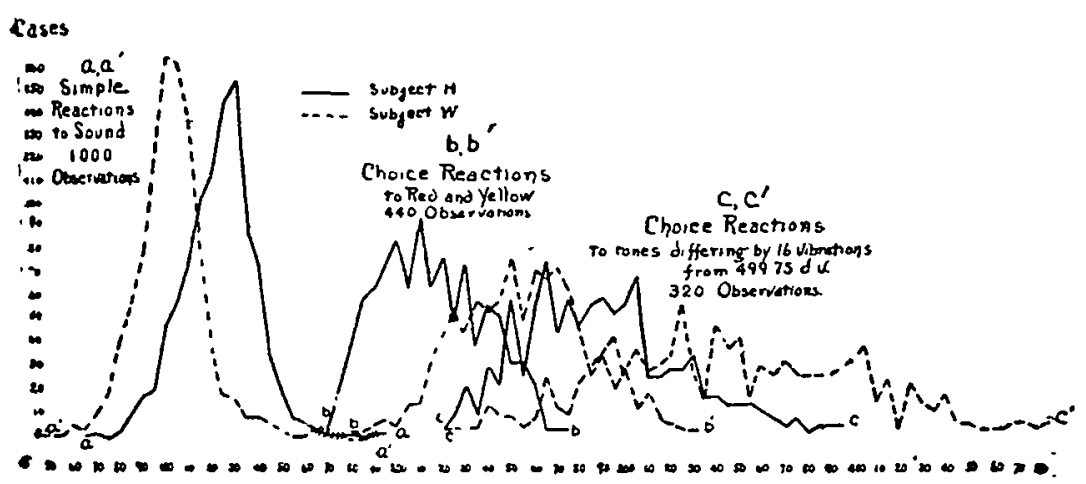

To render the curves more easily comparable, the scale in plotting the choice reactions is altered to make it correspond to that of the simple reactions.

In the two subjects, the simple reaction times differ with the auditory stimuli by $20 \sigma$, with the visual stimuli by nearly I I $\sigma$. Of the fact of these individual differences, preserved long after practice could essentially change them, there can be no dispute. This result is distinctly contrary to the statements of both Alechsieff and Whipple. It raises the question of whether the

1 Froeberg, 'The Reiation between the Magnitude of the Stimulus and the Time of Reaction,' Archioes of Psychol., No. 8, 1907. 
subjects of either of these writers had had sufficient practice to permit of any generalizations concerning the properties of the simple reaction in advanced stages of practise. Neither adduces definite experimental evidence to this effect. The differences here found, are then, due neither to differences in practise, nor to differences in the direction of the attention (sensory or motor), since, so far as it is ever possible to control this factor subjectively, motor reaction alone was employed. The differences represent more fundamental differences in the speed of neural conduction-more probably a difference in the facility of synaptic coördination (or some other interneuronal process) than of transmission along nerve trunks.

Distinct individual differences also exist in the discriminative or choice reactions, but in the opposite direction from those of the simple reactions. Although reacting more quickly to simple light and sound stimuli, $W$ reacts much more slowly where discrimination and choice between two stimuli are involved. The differences in times for the two subjects are $40 \sigma$ for colors and $54 \sigma$ for pitch. It should be noted also that false reactions in the discriminative or choice reactions were throughout nearly twice as frequent in $W$ as in $H$. So far as it was possible to discover (we are now unable to give the exact figures), there was no essential difference in the sensory discriminativeness at the threshold of color or pitch. The significant feature of these results is the relatively greater speed of $W$ in the simple reactions, combined with lower speed in the more complex ones. Since the greater speed of $H$ in these latter reactions does not lie in more open sensori-motor arcs, nor in greater sensory discriminativeness, his results must be referred to more rapid conduction in the higher arcs directly concerned in the organic adjustment to these more complex situations. The independent variability between the simple and compound reactions furnishes strong evidence for the contention that the compound reactions are not merely simple reactions plus such processes as perception, apperception, discrimination, or choice. Hence, the times of these higher mental processes can not be secured, as is so often done, by simple subtraction. 